\title{
Formal and Informal Sectors in a Social Accounting Matrix for Brazil
}

Henrique Morrone*

\begin{abstract}
This paper presents a methodology to estimate a Social Accounting Matrix for Brazil in 2006 that separates between formal and informal sectors. The goal of this study is to estimate and to analyze the Social Accounting Matrix for Brazil in 2006. The shares of output by informal and formal sectors are applied as weights to estimate the size of the two sectors. The results reveal important structural linkages between the two sectors and may serve as data input for future Structuralist Calibrated Models.
\end{abstract}

Keywords: Structuralist calibrated models. Social accounting matrix. Leontief's model. JEL Classification: 017 ; 010 .

\section{Introduction}

One of the increasing concerns in economic development is the measurement of the informal sector in developing economies and its interaction with the formal sector along the cumulative process of growth. Economists agree that in many lowand middle-income countries, the informal sector is a key player as a provider of jobs and a source of labor surplus in periods of rapid output expansion. Moreover, in the 1990s, stylized facts highlight an increase in the share of the informal sector during economic expansion in many developing countries (RADA, 2010). This phenomenon, known as jobless growth, is present in some developing countries such as India, China and in parts of South America.

Because of the critical role of the informal sector in developing economies, it is important to estimate the size of this sector and its relationship with the formal sector as a tool to understand the complexities of the process of economic expansion and to supportfuture economic policy. Policies that try to reduce poverty and promote economic growth must be based on a profound understanding of the economic structure. The lack of reliable statistical data, however, is a significant constraint to achieving a consistent estimation of the Social Accounting Matrix (SAM).

The purpose of this paper is to present the methodology to estimate a Social Accounting Matrix (SAM) that differentiates between formal and informal sectors for Brazil in 2006. The primary source of data used in this dissertation is the national accounting statistics for 2006. Use and resources tables (TRU) were used to build the Input-Output (I-O) table and, consequently, the SAM, in addition to supplementary information from the Flow of Funds (FOF) table for the same year. This study considers the informal sector to be made up of firms that are not

Professor Department of Economic Sciences, Universidade Federal do Rio Grande do Sul. E-mail: hmorrone@hotmail.com 
officially registered with government of Brazil; the informal sector is defined as unorganized activities that present low labor productivity.

There are three main contributions of this paper: First, to present a methodology to build the Input-Output matrix that combines some elements presented in Guilhoto and Sesso (1999) and Grijó and Berni (2006); second, to reveal the structural linkages between the two sectors; third, to offer an estimation of the Social Accounting Matrix that incorporates both formal and informal sectors, serving as a data input for future Structuralist Calibrated models. ${ }^{1}$

This paper is organized as follows. First, after this brief introduction, the basic procedures to harmonize the national accounting tables are presented. Then, the steps of constructing the SAM and its results are documented and analyzed in Section 3. Finally, the last section is reserved for conclusion. The tables used in constructing the FOF part of the Brazilian SAM appear in the appendix.

\section{The Harmonization of National Statistical Accounts}

This section starts with the presentation of the procedure to harmonize the source of data to build a Social Accounting Matrix for Brazil for year 2006. The Social Accounting Matrix provides a schematic behavior of the economy. It describes the circular flow of income inside the economy. The SAM is a union of an Input-Output (I-O) table, which describes the interindustry transactions in the economy, and the flow of funds among institutions. Another characteristic of the SAM is derived from national accounting where expenditure must be equal to income.

Furthermore, the SAM is a square matrix, a necessary condition to the existence of one solution. The columns of the matrix represent purchases while reading across the rows represents sales. The sum of each row must be equal to the sum of each column to guarantee the national accounting condition that income is equal to expenditure.

The SAM has four main building blocks: the Input-Output (I-O) table, the Use of Output table (Final Demand table), the Value-Added table and the Flow of Funds (FOF) table. In the upper west side of the SAM, the Input-Output table describes the transactions among economic activities. For example, the I-O table shows how much the manufacturing sector purchases from mining and quarrying. Next, in the upper east part of the SAM, we have the Use of Output table. This quadrant of the SAM includes five major components: the final consumption by households, government purchases, exports, capital formation and change in stocks. The total

$1 \quad$ Structuralist computable (or calibrated) models consider the structure of the economy and its institutions as important factors to explain the evolution of economic systems. It considers social classes instead of individual behavior; the model usually presents a Keynesian closure. The main reference is Taylor (1983). 
value of output being sold is the result of the addition of the I-O table to the Final Demand table. The quadrant below the I-O table provides the sectoral costs, excluding intermediate inputs, to produce the output being sold. Finally, the Flow of Funds quadrant describes the transfers of income among institutions. This table presents five major institutions: families, government, financial enterprises, nonfinancial enterprises, and the rest of the world. The FOF table is the source of data to build the quadrant in the center of the SAM. Some of the entries in the center of the SAM are: transfer of income from government to workers, income from properties, rents, dividends and interest paid to workers, capital transfers, etc. Table 1 presents the schematic SAM for Brazil with the respective definition for each cell and the origin of the data. The Resources and Uses table (TRU) provides the data to build the I-O table, the Use of Output (Final Demand) table and the Value-Added table. The Integrated Economic Accounts (CEI) is employed to estimate the entries of the FOF table.

Table 1 - A social accounting matrix for a two-sector economy

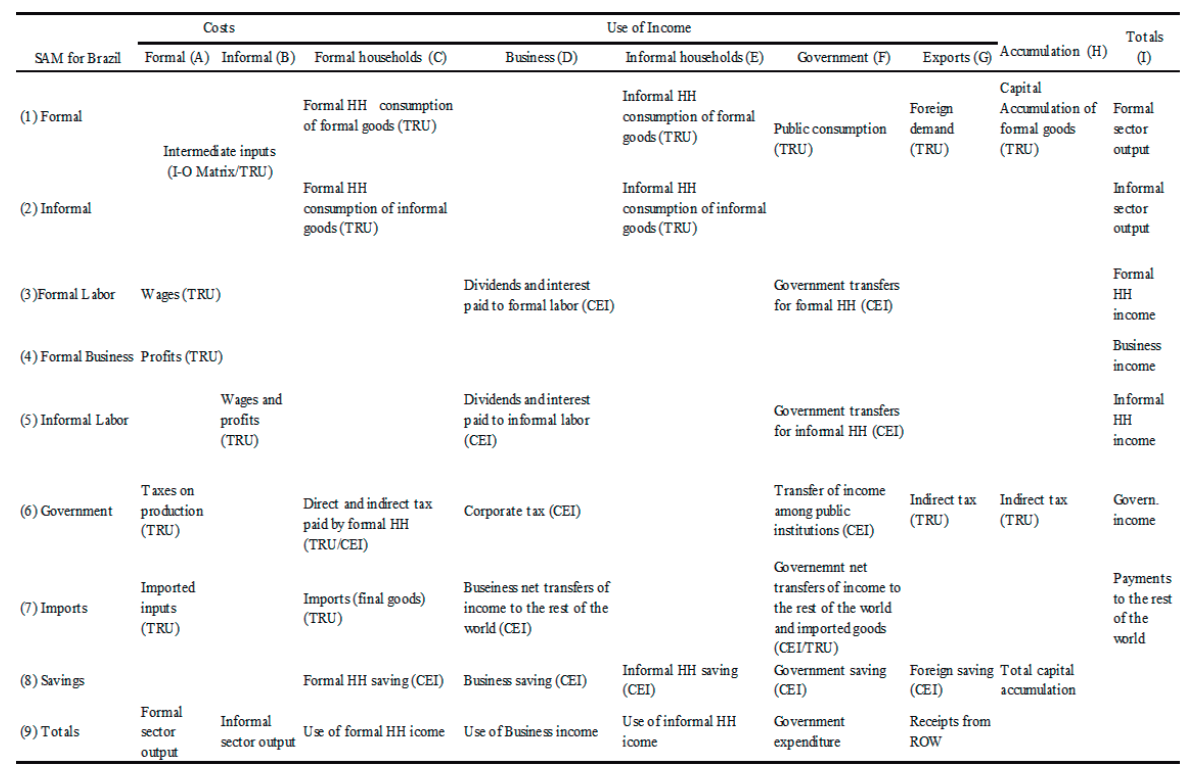

Source: Author's estimations. 
Before we advance in the explanation of the estimation procedures, it is important to emphasize that all the identities presented in the set of equations below were tested to guarantee that the estimation of the I-O table is accurate. For more details see Miller and Blair (1985) and Grijó and Berni (2006).

$$
\begin{aligned}
& q \equiv U n i+f n \\
& B n \equiv U n(1 / \hat{a}) \\
& U n \equiv B n \hat{a} \\
& q \equiv B n a+f n \\
& a \equiv V i \\
& a \equiv D q \\
& a \equiv D(B n a+f n) \\
& a \equiv(I /(I-D B n)) D f n
\end{aligned}
$$

Where:

V: Make matrix;

Un: Use matrix;

$D$ : market-share matrix;

$\mathrm{Bn}$ : technical coefficient matrix of domestic production;

fn: final demand vector;

q: gross production value per good;

a: gross production value per activity;

$\hat{a}$ : gross production value per activity times an identity matrix.

The problem of harmonizing different tables of the national statistical data is derived from the fact that the two major tables (Use ${ }^{2}$ and Make tables), that serve as the main source for the computation of the I-O table, are measured at different prices. To find the I-O table, the Use and Make tables were used. The former represents the demand conditions of the production whereas the latter has the focus on the supply. In other words, the Use table shows the intermediate use of output. Because the Use table originally is measured at market prices, while the Make table is estimated at basic prices, the problem to be solved is to convert the Use matrix into basic prices.

The set of equations below represent the standard procedure to convert the Use table into approximately basic prices. Use and Make tables are released by the Brazilian Institute of Geography and Statistics (SNA- IBGE,) and can be found together in the Resources e Uses table (TRU).

2 Throughout this study the terms 'Use table', 'Intermediate Use of Output table', and 'Intermediate Inputs table' are used interchangeably. 


$$
\begin{aligned}
& S c \equiv S b+C M+T M+T+M \\
& F D \equiv X+G+K+C \\
& T D \equiv D F+I C \\
& T S \equiv T D \\
& S b+C M+T M+T+M \equiv D F+I C \\
& D b \equiv S b \equiv D F+I C-C M-T M-T-M
\end{aligned}
$$

where:

$S c$ : supply measured at consumer prices;

$S b$ : supply of domestic (national) activities measured at basic prices;

$\mathrm{Db}$ : demand measured at basic prices;

$C M$ : transport margins;

$T M$ : trade margins;

$T$ : net taxes;

$M$ : imports;

$F D$ : final demand at consumer prices;

$X$ : exports;

$G$ : government purchases;

$K$ : investment;

$C$ : consumption;

TD : total demand at consumer prices;

IC : intermediate consumption or original use matrix;

TS : total supply at consumer prices.

According to the set of identities above, we may conclude that the solution needed would be to subtract the trade margins matrix, transport margins matrix, and tax and imports matrices from the supply at consumer prices, Sc, to find the supply of national activities measured approximately at basic prices, Sb. Because supply is equivalent to demand, the two components of total demand, the Intermediate Use of Output table and the Final Demand table, might be converted into basic prices simply by a mathematic subtraction. This is exactly the procedure that was applied to convert the Use table into basic prices.

The new Intermediate Use of Output table at basic prices, therefore, will be the result of the subtraction of the trade margins matrix, transport margins matrix, and tax and imports matrices from the original Intermediate Use of Output table, IC, estimated initially at market prices. In the next section, the procedure to estimate the tables is presented in more detail.

Before the complete considerations about the estimation process and its source of data are presented, it is important to reveal some of the basic assumptions of the model and its limitations. The basic limitations of the Leontief model are: 
the presence of constant returns to scale, the classification problem ${ }^{3}$ expressed in the empirical fact that joint products ${ }^{4}$ and by-products ${ }^{5}$ do exist, and the common compromise that occurs every time the Brazilian National Statistical Office releases new data.

In particular, one of the limitations is important here. The classification problem presented in the early version of the Leontief model is solved through the pre-multiplication of the final demand vector, fn, and intersectoral impact matrix, $\mathrm{Bn}$, by the market-share matrix, $D$. For instance, doing the market-share table times the Use table ( $D$ x Un) creates a sector by sector Use matrix. In addition, the technology of the sector is assumed in order to obtain an activity by activity I-O matrix. That is, it assumes that a sector uses the same technology to produce all their goods.

The Input-Output table for Brazil is aggregated into 13 sectors. ${ }^{6}$ Because we do not have the I-O table for 2006, the Input-Output table was derived from national accounting statistics for 2006. The Resources and Uses table provides the complete information needed to construct the I-O table. Furthermore, the data for the Value-Added table and Use of Output table also come from the Resources and Uses table. Lastly, the transfer of funds among institutions is derived from the Flow of Funds table. The same methodology developed by Grijó and Berni (2006) is used. In the next section, the steps to build the I-O table and other results are revealed.

\section{The Complete Methodology to Estimate a SAM for Brazil}

This section describes the methodology used to estimate the SAM for Brazil in 2006. Subsection 3.1 explores the procedures to calculate the I-O table, including the estimation of the main matrices from the previous section. Subsection 3.2 presents the treatment to separate formal and informal activities. Subsection 3.3 presents the two-sector SAM for Brazil. In the appendix, the procedures to calculate some entries of the FOF table, table that reveals the flow of income among institutions, are presented.

3 Problem related with the fact that the A matrix in Leontief's model is not a square matrix but instead it is a commodity by activity matrix. In other words, sectors actually produce and sell a variety of commodities.

4 Two different goods produced simultaneously by the same productive process.

5 Specific productive processes and chemical reactions of some economic activities may generate secondary commodities. These commodities are called by-products. For instance, methane gas (CH4) is a by-product of a chemical reaction that occurs on landfills.

6 These sectors are: agriculture, hunting, forestry and fishing; energy sector, mining and quarrying, manufacturing, public services, construction, wholesale and retail trade, transport and communication, information service, insurance, real estate, other services, and public administration. 


\subsection{From the National Statistical Accounts to the Input-Output Matrix}

The methodology to estimate the Input-Output matrix follows the methodology developed by Guilhoto and Sesso (2002) to build an I-O matrix using national accounting data. According to this work, the tables presented in the previous section (the net tax, the imports, the import tax, the transport and trade margins tables) were estimated and deducted from both Intermediate Use of Output and Final Demand tables. It was needed to convert these two tables into approximately basic prices. In this subsection, we present the procedure of estimation of these tables, including the I-O table. In this sense, the transport and trade margins tables are deducted exclusively from the Intermediate Use of Output table while other important tables such as tax and imports matrices are deducted from both Intermediate Use of Output (Use) and Final Demand matrices.

Using the Resources and Uses table (TRU), ${ }^{7}$ the first challenge is to estimate matrices for the transport margins and trade margins vectors. These matrices must be built because the Intermediate Use of Output table must be measured at basic prices. The procedure is the following. First, each cell of the original Intermediate Use of Output table, Un, should be divided by the gross production value per good, q. The result is a coefficient matrix. Then, multiply the trade and the transport margins vectors by the coefficient matrix to convert the vectors into matrices. Thereafter, subtract the new trade and transport matrices from the Intermediate Use of Output table. This procedure serves to distribute the margins into the Intermediate Use of Output matrix. The assumption is that trade and transport margins are incident only in the input transactions among firms and not in the final demand (see Tables 2 and 3).

$7 \quad$ This table that initially contained 56 economic activities was aggregated into 13 sectors. These sectors are: agriculture, hunting, forestry and fishing; energy sector, mining and quarrying, manufacturing, public services, construction, wholesale and retail trade, transport and communication, information service, insurance, real estate, other services, and public administration. It is assumed that public services and public administration are two formal activities that do not employ informal labor. 
Table 2 - Trade margins for Brazil in 2006

\begin{tabular}{rrrrrrrrrrrrrr}
\hline Sectors & $\mathbf{1}$ & $\mathbf{2}$ & $\mathbf{3}$ & $\mathbf{4}$ & $\mathbf{5}$ & $\mathbf{6}$ & $\mathbf{7}$ & $\mathbf{8}$ & $\mathbf{9}$ & $\mathbf{1 0}$ & $\mathbf{1 1}$ & $\mathbf{1 2}$ & $\mathbf{1 3}$ \\
\hline 1 & 2997,09 & 0,00 & 1035,19 & 18371,56 & 0,00 & 0,00 & 0,00 & 0,00 & 0,00 & 0,00 & 0,00 & 419,41 & 70,76 \\
2 & 134,03 & 359,27 & 3,42 & 2247,39 & 0,00 & 0,00 & 0,00 & 0,00 & 0,00 & 0,00 & 0,00 & 3,33 & 3,42 \\
3 & 1583,81 & 528,97 & 19342,76 & 6817,35 & 1596,65 & 689,30 & 1693,73 & 6266,12 & 54,80 & 134,31 & 98,22 & 835,32 & 1235,17 \\
4 & 14518,69 & 1662,51 & 4797,83 & 160375,09 & 2350,31 & 19417,26 & 4469,10 & 5270,31 & 3617,23 & 2542,07 & 450,98 & 29374,65 & 7427,98 \\
5 & 0,00 & 0,00 & 0,00 & 0,00 & 0,00 & 0,00 & 0,00 & 0,00 & 0,00 & 0,00 & 0,00 & 0,00 & 0,00 \\
6 & 0,00 & 0,00 & 0,00 & 0,00 & 0,00 & 0,00 & 0,00 & 0,00 & 0,00 & 0,00 & 0,00 & 0,00 & 0,00 \\
7 & 0,00 & 0,00 & 0,00 & $-126950,09$ & 0,00 & 0,00 & $-1594,85$ & $-194125,19$ & 0,00 & 0,00 & 0,00 & $-350,87$ & 0,00 \\
8 & 0,00 & 0,00 & 0,00 & 0,00 & 0,00 & 0,00 & 0,00 & 0,00 & 0,00 & 0,00 & 0,00 & 0,00 & 0,00 \\
9 & 0,00 & 0,00 & 0,00 & 0,00 & 0,00 & 0,00 & 0,00 & 0,00 & 0,00 & 0,00 & 0,00 & 0,00 & 0,00 \\
10 & 0,00 & 0,00 & 0,00 & 0,00 & 0,00 & 0,00 & 0,00 & 0,00 & 0,00 & 0,00 & 0,00 & 0,00 & 0,00 \\
11 & 0,00 & 0,00 & 0,00 & 0,00 & 0,00 & 0,00 & 0,00 & 0,00 & 0,00 & 0,00 & 0,00 & 0,00 & 0,00 \\
12 & 0,00 & 0,00 & 0,00 & 0,00 & 0,00 & 0,00 & 0,00 & 0,00 & 0,00 & 0,00 & 0,00 & 0,00 & 0,00 \\
13 & 0,00 & 0,00 & 0,00 & 0,00 & 0,00 & 0,00 & 0,00 & 0,00 & 0,00 & 0,00 & 0,00 & 0,00 & 0,00 \\
\hline
\end{tabular}

Source: Author's estimations.

Table 3 - Transport margins for Brazil in 2006

\begin{tabular}{|c|c|c|c|c|c|c|c|c|c|c|c|c|c|}
\hline Sectors & 1 & 2 & 3 & 4 & 5 & 6 & 7 & 8 & 9 & 10 & 11 & 12 & 13 \\
\hline 1 & 328,33 & 0,00 & 113,40 & 2012,57 & 0,00 & 0,00 & 0,00 & 0,00 & 0,00 & 0,00 & 0,00 & 45,95 & 7,75 \\
\hline 3 & 129,33 & 43,20 & 1579,52 & 556,74 & 130,38 & 56,29 & 138,31 & 511,69 & 4,47 & 10,97 & 8,02 & 68,21 & 100,86 \\
\hline 5 & 0,00 & 0,00 & 0,00 & 0,00 & 0,00 & 0,00 & 0,00 & 0,00 & 0,00 & 0,00 & 0,00 & 0,00 & 0,00 \\
\hline 6 & 0,00 & 0,00 & 0,00 & 0,00 & 0,00 & 0,00 & 0,00 & 0,00 & 0,00 & 0,00 & 0,00 & 0,00 & 0,00 \\
\hline 7 & 0,00 & 0,00 & 0,00 & 0,00 & 0,00 & 0,00 & 0,00 & 0,00 & 0,00 & 0,00 & 0,00 & $-350,87$ & 0,00 \\
\hline 10 & 0,00 & 0,00 & 0,00 & 0,00 & 0,00 & 0,00 & 0,00 & 0,00 & 0,00 & 0,00 & 0,00 & 0,00 & 0,00 \\
\hline 11 & 0,00 & 0,00 & 0,00 & 0,00 & 0,00 & 0,00 & 0,00 & 0,00 & 0,00 & 0,00 & 0,00 & 0,00 & 0,00 \\
\hline 12 & 0,00 & 0,00 & 0,00 & 0,00 & 0,00 & 0,00 & 0,00 & 0,00 & 0,00 & 0,00 & 0,00 & 0,00 & 0,00 \\
\hline 13 & 0,00 & 0,00 & 0,00 & 0,00 & 0,00 & 0,00 & 0,00 & 0,00 & 0,00 & 0,00 & 0,00 & 0,00 & 0,00 \\
\hline
\end{tabular}

Source: Author's estimations.

The procedure to estimate the matrix for net taxes is the following. Each cell of the Intermediate Use of Output table must be divided by the total demand vector. The outcome of this computation is a new coefficient matrix. This new matrix is multiplied by the vector of net taxes to convert it into matrix.

However, the remaining two matrices, imports and import tax matrices, are estimated differently. It is necessary to use a different approach because there is no incidence of imports and imports' taxes in at least one of the final demand components. Imports and imports' taxes should not be deducted from exports. To solve this problem, two specific coefficient matrices are calculated to spread both imports and imports' taxes vectors into the Use and Final Demand matrices.

In this way, the coefficient matrix for the Use table is calculated in two steps. First, the deduction of the exports from the total demand is necessary. Second, each cell of the Use table is divided by the total demand vector (without exports) to get a coefficient matrix that later will be applied to spread imports and import tax into the Intermediate Use of Output (Use) table. Then, the multiplication of the new coefficient matrix by imports and import tax vectors gives the imports and import tax matrices. In short, the five matrices (taxes, trade, transport, imports and 
imports' taxes) are deducted from the Intermediate Use of Output table to convert this table into basic prices.

A Similar procedure is applied into the Final Demand table. More specifically, to estimate the tax matrix, each cell of the final demand matrix is divided by the total demand vector. Consequently, the multiplication of the tax vector by the resulting coefficient matrix produces the tax matrix.

For the same reasons explained previously, the procedure to estimate the remaining matrices is more complex. This time, the coefficient matrix for the Final Demand table is calculated in three steps. Firstly, the deduction of the exports from the total demand is again a necessity. Secondly, the new coefficient matrix must have the whole cells of the column of exports equal zero. Thirdly, each cell of the Final Demand table (deducted of exports) is divided by the total demand vector, a vector that does not include exports, to get a coefficient matrix to spread imports and imports' taxes into the Final Demand matrix. As a result, the simple multiplication of the coefficient matrix by imports and imports' taxes vectors creates the imports and imports' taxes matrices. For more details, see Tables 4-10.

Table 4 - Coefficient matrix

\begin{tabular}{rrrrrrrrrrrrrr}
\hline Sector: & $\mathbf{1}$ & $\mathbf{2}$ & $\mathbf{3}$ & $\mathbf{4}$ & $\mathbf{5}$ & $\mathbf{6}$ & $\mathbf{7}$ & $\mathbf{8}$ & $\mathbf{9}$ & $\mathbf{1 0}$ & $\mathbf{1 1}$ & $\mathbf{1 2}$ & $\mathbf{1 3}$ \\
\hline 1 & 0,0859 & 0,0000 & 0,0297 & 0,5268 & 0,0000 & 0,0000 & 0,0000 & 0,0000 & 0,0000 & 0,0000 & 0,0000 & 0,0120 & 0,0020 \\
2 & 0,0266 & 0,0713 & 0,0007 & 0,4461 & 0,0000 & 0,0447 & 0,0000 & 0,0000 & 0,0000 & 0,0000 & 0,0000 & 0,0007 & 0,0007 \\
3 & 0,0277 & 0,0093 & 0,3388 & 0,1194 & 0,0280 & 0,0121 & 0,0297 & 0,1097 & 0,0010 & 0,0024 & 0,0017 & 0,0146 & 0,0216 \\
4 & 0,0264 & 0,0030 & 0,0087 & 0,2921 & 0,0043 & 0,0354 & 0,0081 & 0,0096 & 0,0066 & 0,0046 & 0,0008 & 0,0535 & 0,0135 \\
5 & 0,0087 & 0,0081 & 0,0245 & 0,2268 & 0,1899 & 0,0029 & 0,0432 & 0,0172 & 0,0127 & 0,0105 & 0,0015 & 0,0836 & 0,0602 \\
6 & 0,0000 & 0,0000 & 0,0090 & 0,0086 & 0,0000 & 0,0196 & 0,0010 & 0,0002 & 0,0035 & 0,0062 & 0,0239 & 0,0163 & 0,0638 \\
7 & 0,0000 & 0,0000 & 0,0000 & 0,3255 & 0,0000 & 0,0041 & 0,4977 & 0,0000 & 0,0000 & 0,0000 & 0,0000 & 0,0009 & 0,0000 \\
8 & 0,0155 & 0,0211 & 0,0579 & 0,1953 & 0,0120 & 0,0060 & 0,0883 & 0,0862 & 0,0179 & 0,0118 & 0,0015 & 0,0461 & 0,0184 \\
9 & 0,0036 & 0,0071 & 0,0189 & 0,0835 & 0,0100 & 0,0026 & 0,0280 & 0,0126 & 0,1543 & 0,0817 & 0,0027 & 0,1910 & 0,1244 \\
10 & 0,0076 & 0,0071 & 0,0075 & 0,1394 & 0,0098 & 0,0078 & 0,0333 & 0,0201 & 0,0179 & 0,1249 & 0,0038 & 0,0241 & 0,1374 \\
11 & 0,0010 & 0,0016 & 0,0257 & 0,0314 & 0,0021 & 0,0025 & 0,0452 & 0,0084 & 0,0212 & 0,0068 & 0,0036 & 0,0374 & 0,0385 \\
12 & 0,0001 & 0,0030 & 0,0188 & 0,0550 & 0,0127 & 0,0068 & 0,0362 & 0,0233 & 0,0293 & 0,0325 & 0,0050 & 0,0610 & 0,0686 \\
13 & 0,0000 & 0,0000 & 0,0000 & 0,0000 & 0,0000 & 0,0000 & 0,0000 & 0,0000 & 0,0000 & 0,0000 & 0,0000 & 0,0000 & 0,0000 \\
\hline
\end{tabular}

Source: Author's estimations.

Table 5 - Net taxes

\begin{tabular}{rrrrrrrrrrrrrr}
\hline Sector: & $\mathbf{1}$ & $\mathbf{2}$ & $\mathbf{3}$ & $\mathbf{4}$ & $\mathbf{5}$ & $\mathbf{6}$ & $\mathbf{7}$ & $\mathbf{8}$ & $\mathbf{9}$ & $\mathbf{1 0}$ & $\mathbf{1 1}$ & $\mathbf{1 2}$ & $\mathbf{1 3}$ \\
\hline 1 & 799,23 & 0,00 & 276,05 & 4899,14 & 0,00 & 0,00 & 0,00 & 0,00 & 0,00 & 0,00 & 0,00 & 111,84 & 18,87 \\
2 & 53,15 & 142,48 & 1,35 & 891,26 & 0,00 & 89,29 & 0,00 & 0,00 & 0,00 & 0,00 & 0,00 & 1,32 & 1,35 \\
3 & 833,82 & 278,48 & 10183,29 & 3589,36 & 840,58 & 362,89 & 891,69 & 3298,89 & 28,85 & 70,71 & 51,71 & 439,77 & 650,28 \\
4 & 4126,95 & 472,57 & 1363,79 & 45586,74 & 668,08 & 5519,37 & 1270,35 & 1498,09 & 1028,20 & 722,59 & 128,19 & 8349,76 & 2111,41 \\
5 & 281,21 & 264,23 & 794,85 & 7356,85 & 6157,26 & 92,83 & 1399,53 & 556,45 & 412,33 & 342,07 & 49,13 & 2710,91 & 1951,46 \\
6 & 0,00 & 0,03 & 50,80 & 48,29 & 0,27 & 110,49 & 5,40 & 1,15 & 19,82 & 34,93 & 134,84 & 91,58 & 359,53 \\
7 & 0,00 & 0,00 & 0,00 & 0,00 & 0,00 & 0,00 & 0,00 & 0,00 & 0,00 & 0,00 & 0,00 & 0,00 & 0,00 \\
8 & 184,73 & 252,28 & 691,75 & 2331,66 & 143,35 & 71,08 & 1054,70 & 1028,86 & 213,36 & 141,09 & 17,80 & 550,20 & 219,87 \\
9 & 121,29 & 235,23 & 628,27 & 2776,99 & 331,53 & 87,56 & 931,14 & 420,48 & 5136,29 & 2720,19 & 88,43 & 6357,18 & 4139,44 \\
10 & 98,06 & 92,29 & 96,51 & 1802,86 & 126,29 & 100,47 & 430,90 & 259,91 & 231,52 & 1615,29 & 48,87 & 311,46 & 1776,07 \\
11 & 1,52 & 2,45 & 39,60 & 48,42 & 3,20 & 3,92 & 69,67 & 12,95 & 32,65 & 10,53 & 5,48 & 57,75 & 59,33 \\
12 & 3,71 & 89,42 & 566,10 & 1654,25 & 380,77 & 205,20 & 1089,57 & 700,94 & 880,59 & 977,87 & 148,92 & 1832,37 & 2060,67 \\
13 & 0,00 & 0,00 & 0,00 & 0,00 & 0,00 & 0,00 & 0,00 & 0,00 & 0,00 & 0,00 & 0,00 & 0,00 & 0,00 \\
\hline
\end{tabular}

Source: Author's estimations. 
Table 6 - Imports for Brazil in 2006

\begin{tabular}{rrrrrrrrrrrrrr}
\hline Sector: & $\mathbf{1}$ & $\mathbf{2}$ & $\mathbf{3}$ & $\mathbf{4}$ & $\mathbf{5}$ & $\mathbf{6}$ & $\mathbf{7}$ & $\mathbf{8}$ & $\mathbf{9}$ & $\mathbf{1 0}$ & $\mathbf{1 1}$ & $\mathbf{1 2}$ & $\mathbf{1 3}$ \\
\hline 1 & 505,58 & 0,00 & 174,63 & 3099,12 & 0,00 & 0,00 & 0,00 & 0,00 & 0,00 & 0,00 & 0,00 & 70,75 & 11,94 \\
2 & 418,11 & 1120,76 & 10,65 & 7010,91 & 0,00 & 702,39 & 0,00 & 0,00 & 0,00 & 0,00 & 0,00 & 10,39 & 10,65 \\
3 & 1104,19 & 368,78 & 13485,25 & 4753,22 & 1113,14 & 480,56 & 1180,82 & 4368,57 & 38,20 & 93,64 & 68,47 & 582,36 & 861,13 \\
4 & 4981,58 & 570,43 & 1646,21 & 55027,13 & 806,43 & 6662,36 & 1533,42 & 1808,32 & 1241,13 & 872,22 & 154,74 & 10078,89 & 2548,65 \\
5 & 22,84 & 21,46 & 64,55 & 597,49 & 500,07 & 7,54 & 113,66 & 45,19 & 33,49 & 27,78 & 3,99 & 220,17 & 158,49 \\
6 & 0,00 & 0,00 & 1,99 & 1,90 & 0,01 & 4,34 & 0,21 & 0,04 & 0,78 & 1,37 & 5,29 & 3,59 & 14,11 \\
7 & 0,00 & 0,00 & 0,00 & 825,32 & 0,00 & 10,37 & 1262,03 & 0,00 & 0,00 & 0,00 & 0,00 & 2,28 & 0,00 \\
8 & 95,53 & 130,46 & 357,72 & 1205,74 & 74,13 & 36,75 & 545,40 & 532,04 & 110,33 & 72,96 & 9,21 & 284,52 & 113,70 \\
9 & 20,53 & 39,81 & 106,34 & 470,02 & 56,11 & 14,82 & 157,60 & 71,17 & 869,34 & 460,40 & 14,97 & 1075,98 & 700,62 \\
10 & 23,08 & 21,73 & 22,72 & 424,41 & 29,73 & 23,65 & 101,44 & 61,19 & 54,50 & 380,25 & 11,50 & 73,32 & 418,10 \\
11 & 13,11 & 21,16 & 341,92 & 418,10 & 27,63 & 33,81 & 601,54 & 111,80 & 281,94 & 90,93 & 47,33 & 498,66 & 512,30 \\
12 & 2,83 & 68,14 & 431,42 & 1260,68 & 290,18 & 156,38 & 830,34 & 534,18 & 671,09 & 745,23 & 113,49 & 1396,43 & 1570,41 \\
13 & 0,00 & 0,00 & 0,00 & 0,00 & 0,00 & 0,00 & 0,00 & 0,00 & 0,00 & 0,00 & 0,00 & 0,00 & 0,00 \\
\hline
\end{tabular}

Source: Author's estimations.

Table 7 - Taxes on imports for Brazil for year 2006

\begin{tabular}{rrrrrrrrrrrrrr}
\hline Sector: & $\mathbf{1}$ & $\mathbf{2}$ & $\mathbf{3}$ & $\mathbf{4}$ & $\mathbf{5}$ & $\mathbf{6}$ & $\mathbf{7}$ & $\mathbf{8}$ & $\mathbf{9}$ & $\mathbf{1 0}$ & $\mathbf{1 1}$ & $\mathbf{1 2}$ & $\mathbf{1 3}$ \\
\hline 1 & 9,34 & 0,00 & 3,23 & 57,28 & 0,00 & 0,00 & 0,00 & 0,00 & 0,00 & 0,00 & 0,00 & 1,31 & 0,22 \\
2 & 0,51 & 1,37 & 0,01 & 8,55 & 0,00 & 0,86 & 0,00 & 0,00 & 0,00 & 0,00 & 0,00 & 0,01 & 0,01 \\
3 & 0,34 & 0,11 & 4,10 & 1,44 & 0,34 & 0,15 & 0,36 & 1,33 & 0,01 & 0,03 & 0,02 & 0,18 & 0,26 \\
4 & 291,58 & 33,39 & 96,36 & 3220,82 & 47,20 & 389,96 & 89,75 & 105,84 & $\mathbf{7 2 , 6 4}$ & 51,05 & 9,06 & 589,93 & 149,18 \\
5 & 0,00 & 0,00 & 0,00 & 0,00 & 0,00 & 0,00 & 0,00 & 0,00 & 0,00 & 0,00 & 0,00 & 0,00 & 0,00 \\
6 & 0,00 & 0,00 & 0,00 & 0,00 & 0,00 & 0,00 & 0,00 & 0,00 & 0,00 & 0,00 & 0,00 & 0,00 & 0,00 \\
7 & 0,00 & 0,00 & 0,00 & 0,00 & 0,00 & 0,00 & 0,00 & 0,00 & 0,00 & 0,00 & 0,00 & 0,00 & 0,00 \\
8 & 0,00 & 0,00 & 0,00 & 0,00 & 0,00 & 0,00 & 0,00 & 0,00 & 0,00 & 0,00 & 0,00 & 0,00 & 0,00 \\
9 & 0,00 & 0,00 & 0,00 & 0,00 & 0,00 & 0,00 & 0,00 & 0,00 & 0,00 & 0,00 & 0,00 & 0,00 & 0,00 \\
10 & 0,00 & 0,00 & 0,00 & 0,00 & 0,00 & 0,00 & 0,00 & 0,00 & 0,00 & 0,00 & 0,00 & 0,00 & 0,00 \\
11 & 0,00 & 0,00 & 0,00 & 0,00 & 0,00 & 0,00 & 0,00 & 0,00 & 0,00 & 0,00 & 0,00 & 0,00 & 0,00 \\
12 & 0,00 & 0,00 & 0,00 & 0,00 & 0,00 & 0,00 & 0,00 & 0,00 & 0,00 & 0,00 & 0,00 & 0,00 & 0,00 \\
13 & 0,00 & 0,00 & 0,00 & 0,00 & 0,00 & 0,00 & 0,00 & 0,00 & 0,00 & 0,00 & 0,00 & 0,00 & 0,00 \\
\hline
\end{tabular}

Source: Author's estimations.

Table 8 - Make matrix

\begin{tabular}{rrrrrrrrrrrrrr}
\hline Sector: & $\mathbf{1}$ & $\mathbf{2}$ & $\mathbf{3}$ & $\mathbf{4}$ & $\mathbf{5}$ & $\mathbf{6}$ & $\mathbf{7}$ & $\mathbf{8}$ & $\mathbf{9}$ & $\mathbf{1 0}$ & $\mathbf{1 1}$ & $\mathbf{1 2}$ & $\mathbf{1 3}$ \\
\hline 1 & 183468 & 128 & 8 & 14963 & 0 & 0 & 8 & 0 & 0 & 0 & 18 & 0 & 0 \\
2 & 0 & 38491 & 20 & 168 & 0 & 25 & 81 & 0 & 0 & 0 & 93 & 0 & 0 \\
3 & 0 & 0 & 235027 & 825 & 0 & 6 & 0 & 0 & 0 & 0 & 475 & 0 & 0 \\
4 & 0 & 3335 & 4572 & 1230853 & 32 & 296 & 380 & 0 & 0 & 0 & 913 & 0 & 0 \\
5 & 0 & 0 & 0 & 0 & 141730 & 0 & 0 & 0 & 0 & 0 & 389 & 60 & 0 \\
6 & 0 & 0 & 0 & 0 & 0 & 180558 & 0 & 0 & 0 & 0 & 606 & 0 & 0 \\
7 & 0 & 0 & 994 & 360 & 0 & 2 & 319130 & 1037 & 369 & 0 & 1455 & 9407 & 0 \\
8 & 0 & 0 & 0 & 0 & 0 & 3 & 84 & 195342 & 0 & 0 & 281 & 6 & 0 \\
9 & 0 & 0 & 0 & 3 & 0 & 0 & -680 & 0 & 150951 & 0 & 207 & 2 & 0 \\
10 & 0 & 0 & 0 & 0 & 0 & 0 & 0 & 0 & 0 & 225857 & 441 & 0 & 0 \\
11 & 0 & 0 & 0 & 2 & 0 & 0 & 61 & 0 & 0 & 0 & 188151 \\
12 & 0 & 0 & 2 & 33 & 0 & 0 & 12889 & 0 & 0 & 0 & 20164 & 479840 \\
13 & 170 & 0 & 16 & 1676 & 2744 & 0 & 1196 & 2236 & 208 & 0 & 654 & 9426 & 455837 \\
\hline
\end{tabular}

Source: Author's estimations. 
Table 9 - Market-share matrix

\begin{tabular}{rrrrrrrrrrrrrr}
\hline Sector: & $\mathbf{1}$ & $\mathbf{2}$ & $\mathbf{3}$ & $\mathbf{4}$ & $\mathbf{5}$ & $\mathbf{6}$ & $\mathbf{7}$ & $\mathbf{8}$ & $\mathbf{9}$ & $\mathbf{1 0}$ & $\mathbf{1 1}$ & $\mathbf{1 2}$ & $\mathbf{1 3}$ \\
\hline 1 & 0,99907 & 0,00305 & 0,00003 & 0,01198 & 0,00000 & 0,00000 & 0,00002 & 0,00000 & 0,00000 & 0,00000 & 0,00008 & 0,00000 & 0,00000 \\
2 & 0,00000 & 0,91746 & 0,00008 & 0,00013 & 0,00000 & 0,00014 & 0,00024 & 0,00000 & 0,00000 & 0,00000 & 0,00043 & 0,00000 & 0,00000 \\
3 & 0,00000 & 0,00000 & 0,97668 & 0,00066 & 0,00000 & 0,00003 & 0,00000 & 0,00000 & 0,00000 & 0,00000 & 0,00222 & 0,00000 & 0,00000 \\
4 & 0,00000 & 0,07949 & 0,01900 & 0,98556 & 0,00022 & 0,00164 & 0,00114 & 0,00000 & 0,00000 & 0,00000 & 0,00427 & 0,00000 & 0,00000 \\
5 & 0,00000 & 0,00000 & 0,00000 & 0,00000 & 0,98079 & 0,00000 & 0,00000 & 0,00000 & 0,00000 & 0,00000 & 0,00182 & 0,00012 & 0,00000 \\
6 & 0,00000 & 0,00000 & 0,00000 & 0,00000 & 0,00000 & 0,99816 & 0,00000 & 0,00000 & 0,00000 & 0,00000 & 0,00283 & 0,00000 & 0,00000 \\
7 & 0,00000 & 0,00000 & 0,00413 & 0,00029 & 0,00000 & 0,00001 & 0,95792 & 0,00522 & 0,00244 & 0,00000 & 0,00680 & 0,01886 & 0,00000 \\
8 & 0,00000 & 0,00000 & 0,00000 & 0,00000 & 0,00000 & 0,00002 & 0,00025 & 0,98352 & 0,00000 & 0,00000 & 0,00131 & 0,00001 & 0,00000 \\
9 & 0,00000 & 0,00000 & 0,00000 & 0,00000 & 0,00000 & 0,00000 & $-0,00204$ & 0,00000 & 0,99619 & 0,000000 & 0,00097 & 0,00000 & 0,00000 \\
10 & 0,00000 & 0,00000 & 0,00000 & 0,00000 & 0,00000 & 0,00000 & 0,00000 & 0,00000 & 0,00000 & 1,00000 & 0,00206 & 0,00000 & 0,00000 \\
11 & 0,00000 & 0,00000 & 0,00000 & 0,00000 & 0,00000 & 0,00000 & 0,00018 & 0,00000 & 0,00000 & 0,00000 & 0,87984 & 0,00006 & 0,00000 \\
12 & 0,00000 & 0,00000 & 0,00001 & 0,00003 & 0,00000 & 0,00000 & 0,03869 & 0,00000 & 0,00000 & 0,00000 & 0,09429 & 0,96204 & 0,00000 \\
13 & 0,00093 & 0,00000 & 0,00007 & 0,00134 & 0,01899 & 0,00000 & 0,00359 & 0,01126 & 0,00137 & 0,00000 & 0,00306 & 0,01890 & 1,00000 \\
\hline
\end{tabular}

Source: Author's estimations.

Table 10 - Use matrix sector by sector for Brazil in 2006 at basic prices

\begin{tabular}{|c|c|c|c|c|c|c|c|c|c|c|c|c|c|}
\hline Sectors & 1 & 2 & 3 & 4 & 5 & 6 & 7 & 8 & 9 & 10 & 11 & 12 & 13 \\
\hline 1 & 14865,16 & 39,63 & 5132,17 & 92554,31 & 46,46 & 386,26 & 93,68 & 104,58 & 71,51 & 50,12 & 8,94 & 2618,30 & 491,12 \\
\hline 3 & 5962,74 & 1988,60 & 72641,39 & 25786,97 & 5998,29 & 2610,39 & 6385,53 & 23536,34 & 219,74 & 510,33 & 371,08 & 3185,98 & 4664,25 \\
\hline 5 & 1229,28 & 1155,49 & 3484,66 & 32165,44 & 26909,21 & 407,08 & 6135,74 & 2436,39 & 1811,87 & 1499,47 & 216,36 & 11865,08 & 8547,07 \\
\hline 6 & 0,60 & 1,93 & 1643,76 & 1566,97 & 9,96 & 3543,19 & 200,41 & 41,82 & 648,05 & 1123,77 & 4324,08 & 2958,09 & 11547,45 \\
\hline 7 & 54,15 & 66,88 & 598,63 & 125636,74 & 166,17 & 1661,61 & 191126,43 & 439,08 & 386,29 & 362,68 & 56,22 & 1122,92 & 790,18 \\
\hline 8 & 3428,19 & 4681,96 & 12843,86 & 43309,42 & 2660,65 & 1320,13 & 19634,61 & 19094,56 & 3965,31 & 2620,22 & 331,44 & 10220,81 & 4091,37 \\
\hline 11 & 185,13 & 298,70 & 4826,04 & 5926,51 & 390,30 & 477,67 & 8526,91 & 1578,49 & 3979,88 & 1284,26 & 668,11 & 7039,44 & 7232,03 \\
\hline 12 & 79,64 & 1456,34 & 9534,71 & 32021,63 & 6106,72 & 3383,69 & 25961,48 & 11333,94 & 14452,54 & 15713,05 & 2443,61 & 29955,15 & 33597,19 \\
\hline 13 & 111,48 & 110,23 & 432,19 & 2577,99 & 679,55 & 138,17 & 1443,18 & 506,25 & 409,64 & 391,97 & 58,43 & 1051,84 & 924,56 \\
\hline
\end{tabular}

Source: Author's estimations.

The Final Demand matrix at approximately basic prices is the result of the final demand at market prices minus the tax matrix, the imports matrix, and the imports' taxes matrix. It is a standard procedure to transform the final demand components previously measured at market prices into basic prices. Table 11 shows the Final Demand matrix (sector by sector) at approximately basic prices. Furthermore, the components of the Use of Output part of the SAM (exports, household consumption, government purchases, and capital accumulation) are the result of these deductions. 
Table 11 - Demand matrix (sector by sector) at approximately basic prices

\begin{tabular}{crrrr}
\hline Sectors & \multicolumn{1}{c}{ Exports } & Government & Consumption & Investment \\
\hline 1 & 23661,24 & 41,71 & 45267,28 & 13160,53 \\
2 & 20091,95 & 0,47 & 407,75 & 1162,48 \\
3 & 28537,89 & 2,30 & 52555,84 & 1375,35 \\
4 & 204203,87 & 3431,29 & 439574,66 & 173546,27 \\
5 & 7,67 & 1,41 & 44296,86 & 9,94 \\
6 & 920,10 & 0,00 & 447,18 & 152186,62 \\
7 & 2711,61 & 222,11 & 7230,46 & 121,83 \\
8 & 4919,27 & 0,14 & 62584,46 & 9,60 \\
9 & 700,84 & 0,06 & 41671,22 & 5,63 \\
10 & 1855,73 & 1553,15 & 100674,94 & 11,07 \\
11 & 2245,53 & 0,71 & 138859,64 & 4724,65 \\
12 & 24541,68 & 11278,42 & 289143,44 & 1924,76 \\
13 & 844,67 & 456063,22 & 8129,33 & 290,29 \\
\hline
\end{tabular}

Source: Author's estimations.

The Input-Output matrix at approximately basic prices, therefore, is the result of the Intermediate Use of Output table at market prices minus the tax matrix, the imports matrix, the import' tax matrix, trade matrix, and transport matrix. In the Appendix A, one of the important components of the Social Accounting Matrix (SAM) is presented. Because the Flow of Funds table, table that measures the flow of income among institutions, is not directly available, an alternative procedure is implemented. Finally, Sections 3.2 and 3.3 present the process of aggregation of the I-O Matrix and SAM, including the methodology that distinguishes between the formal and informal sectors.

\subsection{Formal and Informal Activities in Brazil}

In this study, the informal sector is defined as a subdivision of the household sector in the System of National Accounts - SNA, characterized by a particular way of organizing the production and an unclear division between labor and capital. This sector includes businesses that are not officially registered. Hallak et al. (2010) estimated the size of the informal sector for the aggregate economy and for 10 sectors from 2000 to 2007.Informal labor has two main component parts: autonomous labor and employees without legal contract. Moreover, it is assumed that the informal sector uses only informal labor. To estimate a SAM that separates between formal and informal activities, the estimations by Hallak et al. (2010) 
are used. These estimations for the 10 sectors were disaggregated into 12 sectors following the procedures suggested by the Brazilian Institute of Geography and Statistics (SNA-IBGE, 2009). Table 12, below, presents the 13 shares (weights) for formal and informal sectors.

Table 12 - Sectoral value-added shares

\begin{tabular}{lrr}
\hline Sectors & Informal & \multicolumn{1}{c}{ Formal } \\
\hline Agriculture & 0,505 & 0,495 \\
Mining and Quarrying & 0,027 & 0,973 \\
Energy & 0,000 & 1,000 \\
Manufacturing & 0,062 & 0,938 \\
Construction & 0,265 & 0,735 \\
Wholesale and Retail & 0,210 & 0,790 \\
Transport & 0,227 & 0,773 \\
Information Services & 0,127 & 0,873 \\
Financing and Insurance & 0,009 & 0,991 \\
Real Estate & 0,016 & 0,984 \\
Other Services & 0,227 & 0,773 \\
Distribution of electricity & 0,000 & 1,000 \\
Public Administration & 0,000 & 1,000 \\
\hline
\end{tabular}

Source: Author's estimations.

The statistics of value added for informal activities for the 12 major sectors in 2007 are employed to estimate the shares of the informal and formal sectors in 2006. It is assumed that there is no significant structural change, in terms of the change in the size of the informal sector, between 2006 and 2007. Specifically for agriculture, wage shares for formal and informal sectors are being applied to separate each transaction into four entries. Equations 15 and 16, below, are applied to estimate the shares for the formal and informal sectors for 12 economic activities. The shares are presented below:

$$
\begin{gathered}
\varphi I=\frac{V A I}{V A I+V A F} \\
\varphi F=\frac{V A F}{V A I+V A F}
\end{gathered}
$$


where $\varphi I, \varphi F$ are the shares (weights) for the informal and formal sectors and VAI , VAF are the value-added for the informal and formal sectors, respectively.

Further, these sectoral shares of output are being used as weights to calculate the size of the informal sector. Each recorded transaction in the Input-Output table for the 13 activities will be separated into four entries. This methodology is based in Rada (2010). The four entries for the transaction of intermediate inputs, $X i, j$, purchased by sector $\mathrm{j}$ from sector $\mathrm{i}$ are presented below:

Formal sector i - Formal sector j:

$$
X i, j-F F=X i, j^{*} \varphi F, i^{*} \varphi F, j
$$

Formal Sector i - Informal sector j:

$$
X i, j-F I=X i, j^{*} \varphi F, i^{*} \varphi I, j
$$

Informal sector i - Formal sector j:

$$
X i, j-I F=X i, j * \varphi I, i * \varphi F, j
$$

Informal sector i - Informal sector j:

$$
X i, j-I I=X i, j^{*} \varphi I, i^{*} \varphi I, j
$$

Thereafter, we need to aggregate all the informal sector transactions into a unique informal sector. A similar procedure is adopted to aggregate the set of formal activities into a unique formal sector. The result is an Input-Output table, with only two major sectors, that distinguishes between formal and informal activities.

Slightly different procedure is adopted to separate every transaction in the Use of Output (Final Demand) quadrant of the SAM. The process to separate households between formal and informal is different. For agriculture, this study uses the values of the wage share as weights to divide the consumption between formal and informal households.

To separate the aggregate consumption between the formal and informal for the other remaining sectors, the procedure adopted is to use the previous shares (Table 12), which separate the transactions of the I-O table, and a new one that is the percentage of value-added for the economy as a whole. In this way, the share of the value-added for the formal sector is 78.36 percent, while the informal sector is 21.64 percent. That is, the formal sector represents 78.36 percent of the GDP. The equation below presents the procedure. For instance, the amount of consumption 
that the formal household, $\mathrm{j}$, buys from formal manufacturing, i, is the result of the following equation.

Formal sector i - Household j:

$$
C i, j-F F=C i, j * \varphi F, i * \phi F
$$

where $\phi F$ is the index for the formal sector ( 78.36 percent) and $\varphi F, i$ is the value-added share of the formal sector, in this case manufacturing, used previously as weights to separate informal and formal activities in the Input-Output table. A similar procedure is applied to separate the consumption between formal and informal households for the other 12 sectors. Thereafter, it is possible to aggregate in only two sectors and two consumers. The RAS technique, an algorithm used to balance square matrices, is applied to balance the sum of rows and columns of the SAM.

The Use of Output quadrant of the SAM has three additional components. First, exports are assumed to include formal activities only. The value of 315.24 billion reals represents exports of goods and services together. Next, government purchases are treated as expenditures on formal goods only. The value of 472.59 billion reals describes the government consumption for 2006. Finally, it is assumed that capital accumulation takes place only in the formal sector. The value of 397.03 billion reals, about 16.7 percent of GDP, reveals a low level of investment compared to other fast-growing emerging economies.

The quadrant below the I-O table provides the sectoral costs, excluding intermediate inputs, to produce the output being sold. In this quadrant, the wages of formal and informal labor are presented. Formal workers' remuneration comes directly from national accounting statistics. This group includes the remuneration of employees with legal contracts such as civil servants, military workers, etc.

For informal workers, wages are the result of adding autonomous remuneration $^{8}$ to wages paid to workers in the informal sector. We assume that the informal sector does not employ formal workers. The value of 279.73 billion reals comes directly from national accounting statistics. Profits and wages are put together in the same entry because of the assumption that there is no clear differentiation between labor remuneration and profits for the informal sector. Conversely, for the formal sector, there are two distinct entries for profits and labor remuneration.

Another category of the sectoral costs is the imported inputs. Imported inputs are assumed to be concentrated into the formal sector, that is, only formal activities are capable to import inputs from the rest of the world. Lastly, government tax on production has incidence in the formal sector only. Table 1 shows that the origin of the data comes from the Resources and Uses table (TRU).

8 Autonomous remuneration consists in the remuneration of own-account workers and informal employers. 
Turning now to the center of the SAM, the entries describe the transfers of funds among institutions. Because the Brazilian Statistical Office does not release directly the complete Flow of Funds table, we attempt to estimate the transfers of income among institutions indirectly. The Integrated Economic Accounts (in Portuguese: Contas Econômicas Integradas: $\mathrm{CEI}$ ) provides the main information needed to a reliable estimation of these entries. This table presents five major institutions: families, government, financial enterprises, nonfinancial enterprises, and the rest of the world. Some of the entries in the center of the SAM are: transfer of income from government to workers, income from properties, rents, dividends and interest paid to workers, capital transfers, final goods imports, government and business transfers to the rest of the world, capital goods imports, etc.

There are two major assumptions concerning the numbers in the center of the SAM. First, transfers from government to households and transfer from capitalist to households are calculated endogenously. ${ }^{9}$ Treating transfer as a residual is necessary because of the inconsistencies between different sources of data. The shares of value-added for formal and informal activities are applied as weights to separate transfers between formal and informal workers. Second, informal households do not pay direct (income) tax.

Finally, government savings, capitalist savings, and household savings come from the same table (CEI) and the same code or transaction, B.12. Moreover, the current account result is derived from transaction B.12 (resources). The complete procedure to calculate the remaining entries is described in Appendix A.

\subsection{A Social Accounting Matrix that Includes Formal and Informal Sectors}

The two-sector SAM for Brazil is presented in Table 13. The Input-Output table is located in the northwest corner of the SAM. Equations 17-20 were applied to calculate the I-O table. The formal sector provides inputs to the informal sector in the amount of 169.53 billion Brazilian reals (column B), and provides intermediate goods worth 1,334 billion reals to itself (column A).

9 For instance, the government transfer toward labor is the result of subtracting government spending (excluding transfers) and savings from government revenue. 


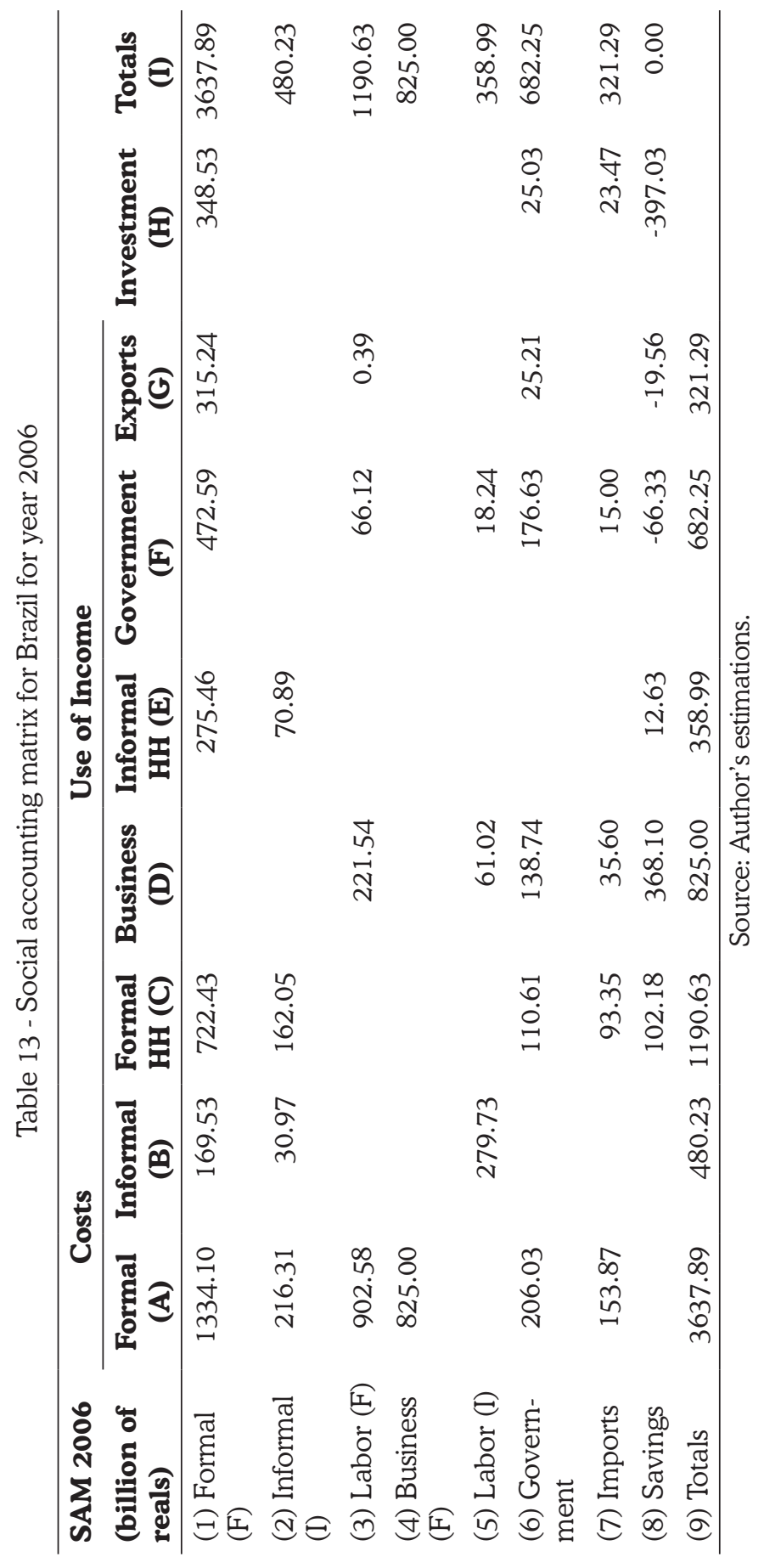

MORRONE, H. Formal and Informal Sectors in a Social Accounting... 
Formal households purchase final goods from the formal sector in the amount of 722.43 billion reals while informal households consume only 275.46 billion reals. Using the classical assumption that capitalist consumption is not significant, the capitalists' consumption is zero (column D).

The amount of 315.24 billion reals (column G) represents the demand from the rest of the world while investment goods (column H) are estimated at 348.53 billion reals. If compared to imports (row 7, column A), equivalent to 153.87 billion reals, the Brazilian economy has a trade surplus in the period. The total output (column I) for the formal sector is estimated at 3,637.89 billion reals. If we add the first row of the I-O table to the first row of the components of the final demand, the total output value can be calculated.

Turning now to the informal sector, similar interpretation can be made. Overall, this sector total output is estimated at 480.23 billion reals. Similar result can be found if we add the Input-Output table (column B) to the cost components (informal compensation). Informal labor compensation captures 279.73 billion reals of the informal sector's output.

Finally, the Flow of Funds table, in the center of the SAM, presents some interesting entries. Formal workers receive wages (column A), transfers from business (dividends, and payment of interest) and government transfers. The total income of formal workers is estimated at 1,190.63 billion reals. In column C, the income of formal household is being spent on 884.48 billion reals of final consumption goods from both sectors.

Informal workers receive wages (column B), transfers from business (dividends, and payment of interest) and government transfers. The total income of informal workers is estimated at 358.99 billion reals. In column $\mathrm{E}$, the income of informal households is being spent on 346.35 billion reals of final consumption goods from both sectors. From this amount, purchases from the informal sector capture 70.89 billion reals, or 20.46 percent of formal household's final demand. For the remaining institutions, other entries can be read in similar fashion.

The results suggest that the informal sector has an important role in the Brazilian economy. Economic policies that intend to reduce poverty and create employment must consider the importance of this sector to the whole economy and its structural relationship with the formal sector.

\section{Final Considerations}

The analyses of the SAM and its components reveal the importance of the informal sector and the relative degree of structural interdependence of the Brazilian economy. Table 14 illustrates important statistics for the two sectors for Brazil in 2006 . 
Table 14 - Summary indicators for Brazil for year 2006

\begin{tabular}{lr}
\hline Economic indicators & \\
\hline Relative labor productivity (formal/informal) & 8.39 \\
Relative wage (formal/informal) & 4.38 \\
Informal employment (\% of total) & 57.59 \\
Saving rate formal HH (\%) & 8.58 \\
Saving rate informal HH (\%) & 3.51 \\
Current account balance/GDP (\%) & -0.97 \\
\hline
\end{tabular}

Source: Author's estimations.

There is a substantial difference in labor productivity between the two sectors. Formal sector workers are on average 8.39 times more productive than workers in the informal sector. The creation of jobs in the formal sector and further increases in productivity are important requirements for sustainable economic growth.

However, to achieve a sustainable process of economic growth, improvements in the labor productivity of informal sector workers are required. The increases in productivity in the informal sector release labor that can migrate toward the formal sector. The transfer of workers from the informal sector, a low labor productivity sector, to the formal sector, a high labor productivity sector, leads to a rise in average labor productivity in the whole economy. This is a precondition for many developing economies that pursue sustainable economic growth.

Turning now to labor remuneration, Table 14 reveals that there is a significant inequality between the two sectors. Labor remuneration in the formal sector on average is about four times higher than labor remuneration in the informal sector. If we consider that labor remuneration in the informal sector includes both capital and labor remunerations, this difference should be even greater.

There are also significant differences in terms of employment indicators. The informal sector employs 53.70 million people, while the formal sector absorbs only 39.54 million people. This illustrates the informal sector's role as a creator of jobs and its capability to absorb surplus labor.

Additionally, it is interesting to make an in-depth analysis of the structural linkages between the two sectors. Table 15 provides the Leontief inverse matrix. The formal sector has the largest impact on the economy through its overall multiplier of 1.74. It means that a unit of increase in the demand of the formal sector good causes the total output to increase 1.74 units. The informal sector has a slightly lower impact on the economy; its overall multiplier is 1.72 . These results suggest that policies that intend to improve economic activity should focus on stimulating demand in both sectors. However, the overall impact of the informal sector on the economy might be overestimated because of the aggregation of he- 
terogeneous subsectors into the informal sector. Rada (2010) points out that further efforts should be made to estimate structural linkages between the formal sector and specific informal subsectors.

Table 15 - The Leontief inverse matrix

\begin{tabular}{lrr}
\hline Sectors & $\mathbf{1}$ & $\mathbf{2}$ \\
\hline (1) Formal & 1.637 & 0.618 \\
(2) Informal & 0.104 & 1.108 \\
Multiplier & 1.741 & 1.726 \\
\hline
\end{tabular}

Source: Author's estimations.

Analyzing the other elements of the Leontief inverse matrix, we can see that the elements of its main diagonal, as expected, are larger than one. The off-diagonal elements, measures of backward linkages between the two sectors, suggest that the informal sector is highly dependent on formal sector provisions of intermediate goods. To satisfy a unit of increase in the demand of the informal commodity, the informal sector needs to demand 0.61 units from the formal sector's good. On the other hand, the formal sector is not very dependent from the informal sector's goods. The formal sector only needs 0.10 units from the informal sector's goods in response to an increase of a unit in its own demand.

To improve economic conditions and stimulate sustainable economic expansion, policies that focus on formal and informal sectors are required. The SAM and its multipliers suggest that the informal sector is important in the Brazilian economy as a generator of jobs and a strategic sector to absorb labor during economic downturns. Policies that try to increase labor productivity in the informal sector are relevant to boost economic growth. Any policy-driven Structuralist Calibrated model, therefore, should consider the intrinsic relationship between the two sectors and the major role that the informal sector has in the process of economic growth.

\section{Referências}

BERNI, D.; LAUTERT, V. Mesoeconomia: lições de contabilidade social. Porto Alegre: Bookman, 2011.661 p.

GRIJÓ, E.; BERNI, D. A metodologia completa para a estimativa de matrizes de insumoproduto. Teoria e Evidência Econômica, Passo Fundo, v. 14, n. 26, p. 9-42, 2006.

GUILHOTO, J. J. M.; SESSO, U. A. Estimação da matriz insumo-produto a partir de dados preliminares das contas nacionais. Economia Aplicada, São Paulo, v. 9, n. 2, p. 277-299, 2005.

HALLAK, J.; NAMIR, K.; KOSOVITS, L. Setor e emprego informal no Brazil: análise dos resultados da nova série do sistema de contas nacionais 2000/2007. In: ENCONTRO 
NACIONAL DE ECONOMIA, 38., 2010, Foz do Iguaçu. Anais... Foz do Iguaçu, ANPEC, 2010. p. 1-19.

IBGE. 1994-2009. Tabela de Recursos e Usos. Disponível em: <http:/www.ibge.gov.br/ home/estatistica/economia/contasnacionais/2009 > . Access in: Sept. 8, 2010.

JORGENSON, D. W. Surplus agricultural labor and the development of a dual economy. Oxford University Papers, Oxford, v. 19, p. 288-312, 1967.

JORGENSON, D. W. The development of a dual economy. The Economic Journal, Oxford, v. 71, n. 282, p. 309-334, 1961.

LEONTIEF, W. Input-output economics. New York: Oxford University Press, 1986. 436 p.

LEWIS, W. A. Economic development with unlimited supplies of labour. Manchester School, Manchester, v. 22, n. 2, p. 139-191, 1954.

MILLER, R. E.; BLAIR, P. D. Input-output analysis: foundations and extensions. Englewood Cliffs: Prentice-Hall, 1985. 750 p.

PYATT, G. A SAM approach to modeling. Journal of Policy Modeling, England, v. 10, n. 3, p. 327-352, 1988.

PYATT, G. Fundamentals of social accounting. Economic Systems Research, Oxford, v. 3, n. 3, p. 315-341, 1991.

RADA, C. Formal and informal sectors in China and India. Economic Systems Research, Oxford, v. 22, n. 2, p. 129-153, 2010.

RADA, C. Stagnation or transformation of a dual economy through endogenous productivity growth. Cambridge Journal of Economics, Oxford, v. 31, n. 5, p. 711-740, 2007.

ROS, J. Development and the economics of growth. Ann Arbor: The University of Michigan Press, 2000. 448 p.

TAYLOR, L. Macro models for developing countries. New York: McGraw-Hill, 1979. 259 p.

TAYLOR, L. Structuralist macroeconomics: applicable models for the third world. New York: Basic Books, 1983. 234 p.

THOMAS, V. B. Input-output analysis in developing countries: sources, methods and applications. John Wiley and Sons, 1982. 314 p.

\section{Appendix A - The Flow of Funds Table}

Because the Brazilian Statistical Office does not directly release the complete Flow of Funds table, we attempt to estimate the transfers of resources among institutions indirectly. The Integrated Economic Accounts (CEI) in 2006 provides the main information needed to a reliable estimation of the transfers of income among institutions. We follow Grijó and Berni (2006) methodology to estimate the flow 
of funds. We present only the tables' results to save space. For further details, see Grijó and Berni (2006).

Table 16 - Economic transfers among institutions (billions of reals)

\begin{tabular}{|c|c|c|c|c|c|c|c|c|c|c|}
\hline \multirow[b]{2}{*}{ Total } & \multicolumn{4}{|c|}{ USES } & \multirow[b]{2}{*}{ Code/Transaction } & \multicolumn{5}{|c|}{ RESOURCES } \\
\hline & ROW & Families & Government & Business & & Business & Government & Families & ROW & Total \\
\hline \multirow[t]{5}{*}{1507,29} & 14,47 & 66,87 & 248,63 & 1177,32 & D. 4 & 1069,87 & 135,04 & 228,94 & 73,45 & 1507,29 \\
\hline & 12,38 & 66,73 & 248,63 & 931,93 & D.41 & 996,81 & 104,03 & 123,08 & 35,76 & \\
\hline & 2,09 & & & 183,10 & D. 42 & 70,97 & 10,64 & 65,88 & 37,69 & \\
\hline & & & & 42,19 & D. 44 & 2,09 & 0,13 & 39,97 & & \\
\hline & & 0,14 & & 20,11 & D. 45 & & 20,25 & & & \\
\hline \multirow[t]{4}{*}{286,11} & 10,54 & 28,42 & 206,01 & 41,13 & D.7 & 28,99 & 208,60 & 47,34 & 1,18 & 286,11 \\
\hline & & 5,62 & 0,09 & 3,35 & D.71 & 9,06 & & & & \\
\hline & & & & 9,06 & D.72 & 3,30 & 0,03 & 5,73 & & \\
\hline & 10,35 & 22,81 & 18,01 & 26,04 & D.75 & 4,76 & 29,93 & 41,60 & 0,91 & \\
\hline 40,72 & & & 15,90 & 24,82 & D. 8 & & & 40,72 & & 40,72 \\
\hline 1834,12 & 25,01 & 95,30 & 470,54 & 1243,27 & TOTAL & 1098,86 & 343,65 & 316,99 & 74,62 & 1834,12 \\
\hline 271,30 & & & 126,89 & 144,41 & NET RESULT & & & 221,69 & 49,61 & 271,30 \\
\hline
\end{tabular}

Source: Contas Econômicas Integradas (CEI/BGE) e author's estimations.

Table 17 - Economic transfers among institutions (billions of reals)

\begin{tabular}{|c|c|c|c|c|c|c|c|c|c|c|}
\hline \multicolumn{5}{|c|}{ USES } & \multirow[b]{2}{*}{ Code/Transaction } & \multicolumn{5}{|c|}{\begin{tabular}{|l} 
RESOURCES \\
\end{tabular}} \\
\hline Total & ROW & Families & Government & Business & & Business & Government & Families & ROW & Total \\
\hline & & & 144,61 & & D. 41 & 64,89 & & 56,35 & 23,37 & \\
\hline & & & & 112,13 & D. 42 & & 10,64 & & 35,60 & \\
\hline & & & & 40,10 & D.44 & & 0,13 & 39,97 & & \\
\hline & & 0,14 & & 20,11 & D.45 & & 20,25 & & & \\
\hline & & 5,62 & 0,09 & & D.71 & 5,71 & & & & \\
\hline & & & & 5,76 & D.72 & & 0,03 & 5,73 & & \\
\hline & & & 0,08 & & D.74 & & & & 0,08 & \\
\hline & 9,44 & & & 21,27 & D.75 & & 11,92 & 18,80 & & \\
\hline
\end{tabular}

Source: Contas Econômicas Integradas (CEI/IBGE) e author's estimations.

Table 18 - Economic transfers among institutions (billions of reals)

\begin{tabular}{crr|c|cc}
\hline \multicolumn{2}{c|}{ USES } & & \multicolumn{2}{c}{ RESOURCES } \\
Total & Government & Business & CODE / TRANSACTION & ROW & Total \\
\hline 23,37 & 23,37 & \multirow{2}{*}{35,60} & D. 41 & 23,37 & 23,37 \\
35,60 & & D.42 & 35,60 & 35,60 \\
& & & D.71 & & \\
& & D.72 & & \\
0,08 & 0,08 & D.74 & 0,08 & 0,08 \\
$-9,44$ & $-9,44$ & D.75 & $-9,44$ & $-9,44$ \\
& & & Net income transfer to rest & & \\
49,61 & 14,01 & 35,60 & of the world & 49,61 & 49,61 \\
\hline
\end{tabular}

Source: Contas Econômicas Integradas (CEI/BGE) e author's estimations. 
Recebido em: 16/10/2014.

Aceito em: 12/02/2015. 